\title{
Heterogeneous Multi-Layer Access and RRM for the Internet of Things
}

\author{
Jia Shen ${ }^{\# 1}$, Xiangyou Lï ${ }^{* 2}$, Huafei $\mathrm{Li}^{* 3}$, Fei Xü${ }^{\# 4}$ \\ ${ }^{*}$ Radio \& Mobile Department, Research Institute of Telecommunications Transmission, MIIT of China \\ NO.11, Yue Tan Nan Jie, Xicheng District, Beijing, 100045, CHINA \\ ${ }^{1}$ shenjia@mail.ritt.com.cn \\ ${ }^{4}$ xufei@mail.ritt.com.cn \\ "School of information and Communication Engineering, Beijing University of Post and Telecommunications \\ Xi Tu Cheng Road, Haidian District, Beijing, 100876, CHINA \\ ${ }^{2} 02$ jordan@bupt.cn \\ ${ }^{3} 1$ hf 4525 @bupt.cn
}

\begin{abstract}
This paper first introduces a heterogeneous IoT access network architecture for the Internet of Things (IoT). In the network, an IoT terminal can adaptively access to a wireless sensor network (WSN) gateway or a cellular base station (BS) according to the channel conditions. The heterogeneous IoT access network schedules the IoT terminal to access to either the WSN or the cellular network $(\mathrm{CN})$ via a joint scheduling RRM algorithm. The simulations show that the heterogeneous IoT system provides a capacity gain over the simple combination of WSN and CN.
\end{abstract}

Keywords - the Internet of the Things, machine-to-machine, heterogeneous network, resource management.

\section{INTRODUCTION}

The Internet of Things (IoT) is a new concept compared to The traditional Internet is used to communicate people (i.e. human-to-human $(\mathrm{H} 2 \mathrm{H})$ communications). Different from the Internet of Human, IoT is generally used for machine-tomachine (M2M) communications. Therefore an important topic of IoT study would be how to enable an efficient and economic M2M communications.

A traditional M2M communications approach is known as "Wireless Sensor Network" (WSN). A WSN has a set of important characteristics: (1) Access in manner of "Ad Hoc"; (2) Networking in manner of "multi-hop" and "Mesh"; (3) Communications between nodes using short-range access techniques, e.g. Wi-Fi, ZigBee, Bluetooth. However, a WSN has a shortage of coverage. Although the coverage of WSN can be extended through multi-hop Mesh network, a WSN can only cover a very limited range.

Therefore an IoT cannot be constructed merely with WSNs. A real IoT would be a convergence of WSN and other widearea communications systems, e.g. cellular networks $(\mathrm{CN})$. The resulting IoT system is a multi-layer heterogeneous access system.

WSN and $\mathrm{CN}$ in general are deployed in different spectrum bands. A WSN usually works with an unlicensed spectrum, e.g. the $2.4 \mathrm{GHz}$ band. While a $\mathrm{CN}$ uses a licensed spectrum, especially a relatively low frequency band, e.g. the 800 $900 \mathrm{MHz}$ band.
Different access techniques and different spectrums provide different coverage ranges. A WSN usually provides a "hotspot" coverage, while a CN provides a full coverage. Therefore the problem is how to select a more suitable access technique and a more suitable spectrum for an IoT terminal according to its position and channel environment.

Obviously, An IoT terminal outside the WSN area can only access to the CN. However, an IoT terminal in the WSN area can access to either the WSN or the $\mathrm{CN}$. Hence the access resource of WSN and that of $\mathrm{CN}$ have different values. The $\mathrm{CN}$ resource has a much higher value than the WSN resource. Meanwhile, as well known, the cost of the $\mathrm{CN}$ resource is also higher than the WSN resource.

Therefore, a more effective and reasonable sharing of the resources is necessary. The principle of the algorithm is to first allocate the WSN (low-value and low-cost) resource to the terminals that can use it (i.e. if they are located in the WSN area). The $\mathrm{CN}$ resource (high-value and high-cost) is saved for the terminals that cannot use the WSN resource (i.e. if they are located outside the WSN area).

Some economic approaches (e.g. Game Theory) are introduced for the sharing between spectrums with different qualities [1-5]. These schemes set different "prices" for the resources with different "values". The higher the value, the higher the price.

In this paper, we propose a resource management scheme with a principle similar to "Pricing" schemes. Using this scheme, an IoT system "auctions" the $\mathrm{CN}$ resource to terminals that like to take higher prices, while "sells" the WSN resource to the terminals that only like to take lower prices. This approach actually attracts those terminals in the WSN area to "buy" the WSN resource with a cheaper price. Compared to the traditional scheme in which the WSN and the $\mathrm{CN}$ resources are allocated separately (auctioned with equal prices), the proposed scheme can obtain capacity gain because of the better usage of $\mathrm{CN}$ resources.

The rest of the paper is organized as following: Section II describes the multi-layer heterogeneous IoT network architecture we consider in the paper. In Section III, the crosslayer resource management scheme is introduced, including how to schedule a terminal to a suitable band. In Section IV, 
the capacity performance comparison between the proposed scheme and the traditional scheme is demonstrated with simulation results. Section V finally concludes the paper.

\section{HETEROGENEOUS IOT NETWORK ARCHITECTURE}

The Internet of Things (IoT) is generally used for machineto-machine (M2M) communications. Especially, the IoT terminals are usually sensors and automatic machines and instruments. Hence the IoT terminals need to be deployed in all kinds of positions and environments.

However, a traditional Wireless Sensor Network (WSN) cannot provide a full coverage. A WSN is usually based on some short-range access techniques, e.g. Wi-Fi, ZigBee, Bluetooth. These techniques only support coverage of 10$100 \mathrm{~m}$. Although the coverage of WSN can be extended through multi-hop Mesh network, a WSN can only cover a very limited range.

A typical WSN topology is shown in Fig. 1. Some IoT terminals (sensor) can be connected to IoT directly via wired link (named "Hop 1" nodes). The IoT terminals that cannot be wired connected first communicate with the Hop 1 nodes via WSN radio. The Hop 1 nodes transfer the data from the Hop 2 nodes. In other words, a IoT terminal can be connected to the IoT in a manner of "multi-hop".

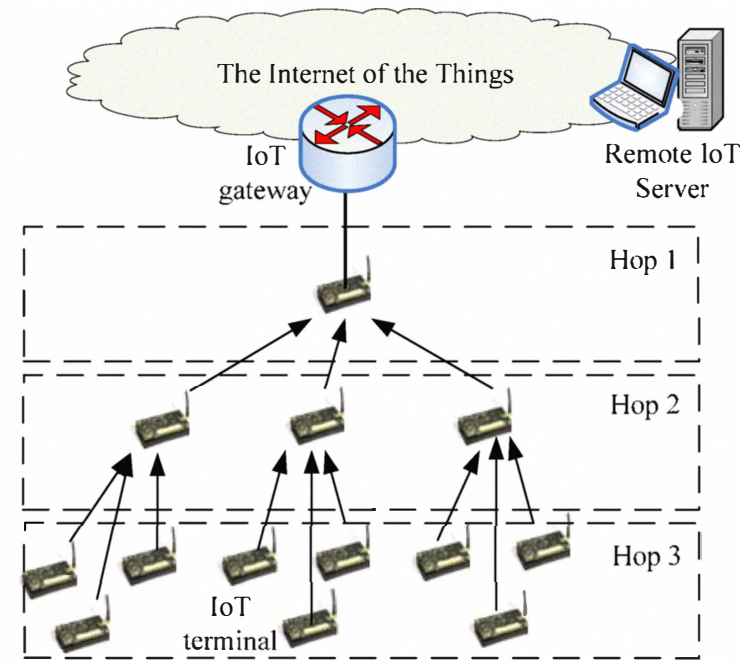

Fig. 1. Traditional WSN topology

However, the number of hops is not unlimited. If a WSN is constructed with too many hops, the lower-hop nodes will take increasing data throughput and power assumption. Considering an IoT terminal should be a low-cost, simple device, only a small number of hops are allowed. If the number of hops is limited (e.g. 3-4 hops), the coverage of a WSN is still very limited.

Therefore an IoT cannot be constructed merely with WSNs. A real IoT would be a convergence of WSN and other widearea communications systems, e.g. cellular networks (CN). A $\mathrm{CN}$ provide a wide-area coverage (normally $90 \%$ ). If an IoT supports the $\mathrm{CN}$ radio, it can access to the network wherever it is. Furthermore, WSN and $\mathrm{CN}$ in general are deployed in different spectrum bands. A WSN usually works with an unlicensed spectrum, e.g. the $2.4 \mathrm{GHz}$ band. While a $\mathrm{CN}$ uses a licensed spectrum, especially a relatively low frequency band, e.g. the $800-900 \mathrm{MHz}$ band.

Hence both WSN and CN should be jointly used for the IoT. The resulting IoT system is a multi-layer heterogeneous access system, as shown in Fig. 2.

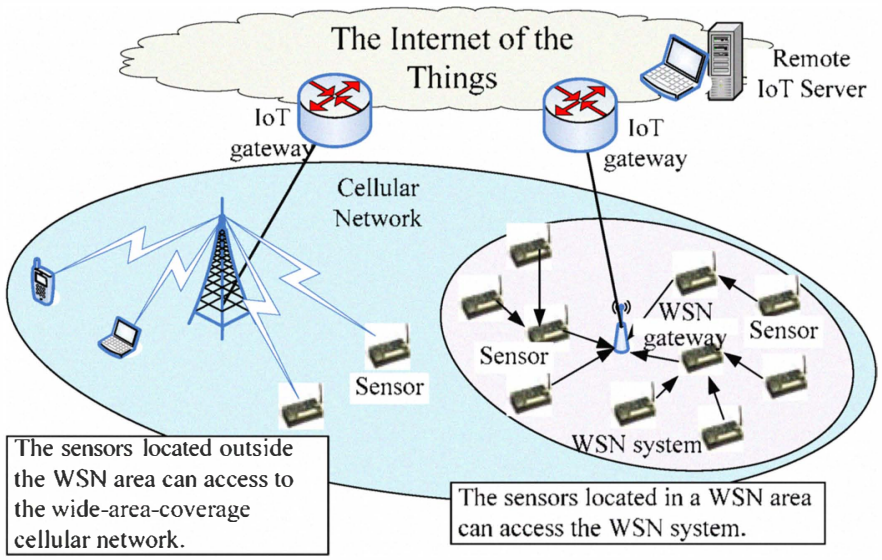

Fig. 2. Heterogeneous access system for IoT

In the system shown in Fig. 2, the IoT terminal in the WSN coverage area (white area) can access to either the WSN or $\mathrm{CN}$. The IoT terminal outside the WSN coverage can only access to the $\mathrm{CN}$ system (blue area). Hence the access resource of WSN and that of $\mathrm{CN}$ have different values. The $\mathrm{CN}$ resource has a much higher value than the WSN resource. Meanwhile, as well known, the cost of the $\mathrm{CN}$ resource is also higher than the WSN resource.

A CN uses expensive licensed spectrum. And many other types of terminals, e.g. mobile phones, laptop computers need to be served (usually with a higher priority than IoT terminals). A WSN uses free unlicensed spectrum, and is only used to support a limited number of IoT terminals.

In this case, the problem is how to select a more suitable access technique and spectrum for a specific IoT terminal by balancing between the value and the cost of the resources.

The principle of the algorithm is to first allocate the WSN (low-value and low-cost) resource to the terminals that can use it (i.e. if they are located in the WSN area). The $\mathrm{CN}$ resource (high-value and high-cost) is saved for the terminals that cannot use the WSN resource (i.e. if they are located outside the WSN area). Another selection metric is mobility. If a terminal has a high mobility, even though it is located in the WSN area, it can hardly access the WSN system (WSN radio cannot well support high mobility).

In order to deeply study and simulate the system, we here consider a simplified heterogeneous network architecture. Fig. 3 illustrates an example of the dual-layer (considering the simplest scenario) IoT network. The WSN gateways and the $\mathrm{CN}$ base stations (BS) are co-located while the coverage of WSN is smaller than that of CN. Only one-hop WSN is considered for simplicity. 


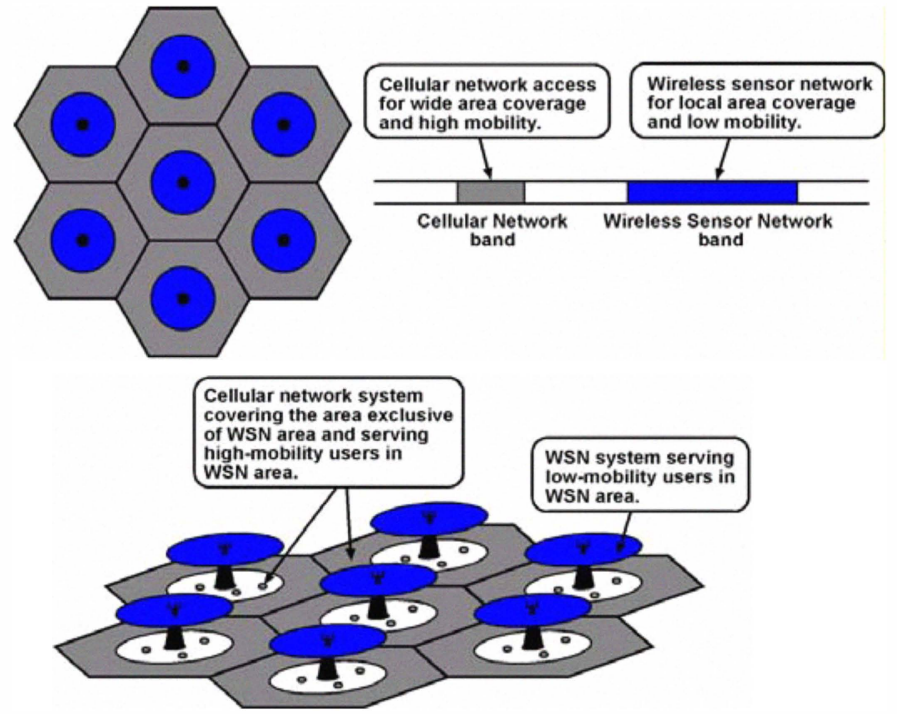

Fig. 3 Dual-layer heterogeneous IoT network architecture

This network architecture consists of two layers: WSN layer and $\mathrm{CN}$ layer. If an IoT terminal is located in the coverage of the WSN layer and with a low mobility, the terminal is scheduled to the WSN layer. If a terminal is located outside the coverage of the WSN layer or with a high mobility, the terminal is scheduled to the $\mathrm{CN}$ layer.

\section{MULTI-LAYER RESOURCE MANAGEMENT}

The WSN layer provides a smaller coverage range than the $\mathrm{CN}$ layer, hence only covers the centre area of the cells (as shown in Fig. 3). The CN layer is used to serve the cell-edge terminals and the high-mobility terminals.

As shown in Fig. 4, the multi-layer RRM operation in the two layers are not realized in an independent manner, but performed jointly. The terminals are scheduled cross the two layers based on a "joint scheduling" operation, following the criteria below:

- If a terminal is located in the WSN area, and with a low mobility, it is served with the WSN layer.

- If a terminal is located outside the WSN area, it is served with the $\mathrm{CN}$ layer.

- If a terminal is located in the WSN area, but with a high mobility, it is served with the $\mathrm{CN}$ layer.

The WSN covers the "cell-centre" area and fixed/lowmobility terminals. Hence the $\mathrm{CN}$ can concentrate its "precious" frequency resource on "cell-edge" area and highmobility terminals, and a higher system throughput can be achieved in cell edge area. The load balancing between the two layers are supported for efficient use of access resources.

The joint scheduling procedure is demonstrated in Fig. 4.

(1) Terminal measures the signal qualities of WSN gateways and $\mathrm{CN}$ BSs respectively.

(2) Terminal calculates the channel quality indicator (CQI) for both the two layers based on the measurements.

(3) Terminal reports the two CQIs to the IoT system scheduler.
(4) The IoT system scheduler compares the CQIs of the two layers. Before compared, the WSN's CQI is added to an extra weight (to set a lower price to WSN).

(5) The IoT system scheduler makes the scheduling decision, and informs it to the terminal.

(6) The terminal raises an access attempt to the selected layer.

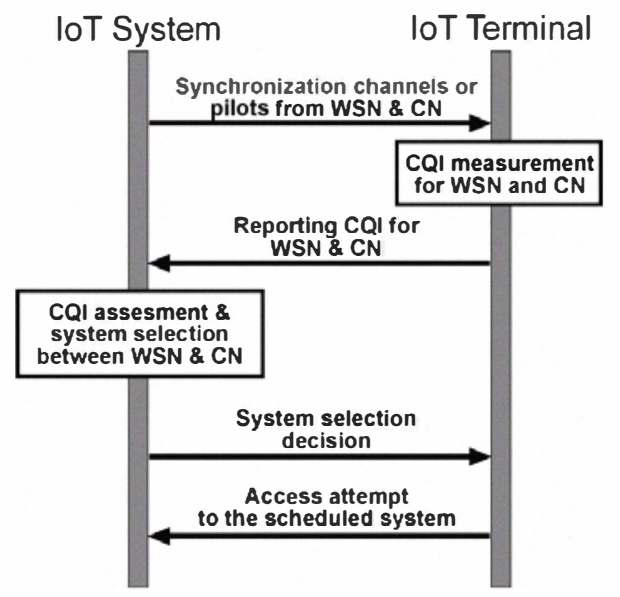

Fig. 4. Multi-layer RRM for heterogeneous IoT system

It should be noted that the above signalling procedure should be carried in $\mathrm{CN}$ layer, because the terminal is not necessarily located in WSN area.

\section{SIMULATION RESULTS AND ANALYSIS}

In this section, we simulate the performance of the proposed multi-layer scheduling scheme. We consider a 3GPP LTE (Long-Term Revolution) system with the 19-BS 57-cell layout (each BS contains 3 cells (sectors)) as the $\mathrm{CN}$ layer. $2.4 \mathrm{GHz}$ IEEE $802.11 \mathrm{~g}$ is considered as the WSN layer. The downlink spectrum efficiency (bit/s/Hz/cell) of the center cell is simulated. In each cell, the WSN gateway (Wi-Fi AP) and the CN BS (LTE eNodeB) are co-located (just as shown in Fig. 3).

Since the CN has a better coverage than WSN. The SINR of a terminal at the edge of the $\mathrm{CN}$ cell will be much larger than the WSN cell. Each cell has $10 \mathrm{MHz}$ spectrum for WSN layer and $10 \mathrm{MHz}$ for $\mathrm{CN}$ layer. The LTE eNodeBs employ the closed-loop precoding spatial multiplexing (Transmission Mode 4 in [6-7]) in downlink transmission. The carrier frequency of $\mathrm{CN}$ is set to $800 \mathrm{MHz}$, and that of WSN is set to $2.4 \mathrm{GHz}$. We drop 20 terminals in a cell (the cell uses WSN and $\mathrm{CNs}$ ). It is assumed that the WSN and the $\mathrm{CN}$ can serve up to 10 terminals respectively. Each layer will try to serve as many terminals as possible. However, if over 10 terminals try to access to any layer of the two, the layer will select the 10 terminals with the highest SINRs, while reject the other terminals.

The simulation parameters are summarized in Table I [8].

TABLE I

SIMULATION ASSUMPTIONS

\begin{tabular}{|l|l|}
\hline Parameter & Value \\
\hline Number of Cells & 19 \\
\hline
\end{tabular}




\begin{tabular}{|c|c|c|}
\hline \multicolumn{2}{|c|}{ Number of Sectors per Cell } & 3 \\
\hline \multicolumn{2}{|c|}{ Inter-site distance $(\mathrm{m})$} & 1732 \\
\hline \multicolumn{2}{|c|}{ Antenna Configuration } & $4 \times 2$ \\
\hline \multicolumn{2}{|c|}{ Antenna type } & Independence \\
\hline \multicolumn{2}{|c|}{ Centre Frequency $(\mathrm{CN})$} & $800 \mathrm{MHz}$ \\
\hline \multicolumn{2}{|c|}{ Centre Frequency (WSN) } & $2.4 \mathrm{GHz}$ \\
\hline \multicolumn{2}{|c|}{ Traffic model } & Full Buffer \\
\hline \multicolumn{2}{|c|}{ BS/AP transmit power } & $46 \mathrm{dBm}$ \\
\hline \multicolumn{2}{|c|}{ Lognormal Shadowing } & Standard deviation: $10 \mathrm{~dB}$ \\
\hline \multirow[t]{2}{*}{$\begin{array}{l}\text { Shadowing } \\
\text { Correlation }\end{array}$} & $\begin{array}{l}\text { Between } \\
\text { cells }\end{array}$ & 0.5 \\
\hline & $\begin{array}{l}\text { Between } \\
\text { sectors }\end{array}$ & 1 \\
\hline \multicolumn{2}{|c|}{ Terminal Noise Figure } & $9 \mathrm{~dB}$ \\
\hline \multicolumn{2}{|c|}{ Terminal Antenna Gain } & $0 \mathrm{dBi}$ \\
\hline \multicolumn{2}{|c|}{ BS/AP Antenna Gain } & $14 \mathrm{dBi}$ \\
\hline \multicolumn{2}{|l|}{ Path-Loss } & $128.1+37.6 \log 10(\mathrm{R}), \mathrm{R}$ in $\mathrm{km}$ \\
\hline \multicolumn{2}{|c|}{ Thermal Noise Density } & $-174 \mathrm{dBm} / \mathrm{Hz}$ \\
\hline \multicolumn{2}{|c|}{$\begin{array}{l}\text { Antenna Bore-sight points } \\
\text { toward flat side of cell (for } \\
\text { 3-sector sites with fixed } \\
\text { antenna patterns) }\end{array}$} & \\
\hline \multicolumn{2}{|c|}{$\begin{array}{l}\text { BS/AP Antenna } \\
\text { pattern(horizontal) } \\
\text { (For 3-sector cell sites with } \\
\text { fixed antenna patterns) }\end{array}$} & $\begin{array}{l}A(\theta)=-\min \left[12\left(\frac{\theta}{\theta_{3 d B}}\right)^{2}, A_{m}\right] \\
\theta_{3 d B}=70 \text { degrees, } A_{m}= \\
20 \mathrm{~dB}\end{array}$ \\
\hline \multicolumn{2}{|c|}{$\begin{array}{l}\text { Minimum distance between } \\
\text { UE and Cell }\end{array}$} & $>=35$ meters \\
\hline \multicolumn{3}{|c|}{$\begin{array}{l}\text { Terminals dropped } \\
\text { uniformly in entire cell }\end{array}$} \\
\hline \multicolumn{2}{|c|}{ Terminal number per sector } & $10 \sim 20$ \\
\hline \multicolumn{2}{|c|}{ L2S interface } & EESM \\
\hline
\end{tabular}

Two terminal scheduling schemes are compared:

- Scheme 1 (traditional scheme): Each terminal first measures SINR of CN and that of WSN respectively. Then the terminal always selects and tries to access the layer with the higher SINR.

$$
\text { System }=\left\{\begin{array}{cl}
W S N, & \text { if } S I N R_{W S N} \geq S I N R_{C N} \\
C N, & \text { if } S I N R_{W S N}<S I N R_{C N}
\end{array}\right.
$$

- Scheme 2 (proposed scheme): Each terminal first measures SINR of $\mathrm{CN}$ and that of WSN respectively. Then the terminal will add a "Price" weight $W_{\text {Price }}$ to $S I N R_{W S N}$ :

$$
\operatorname{SINR}_{W S N}{ }^{\prime}=S I N R_{W S N}+W_{\text {Price }}
$$

Then the terminal selects and tries to access to the better band with the higher SINR, while using SINR $_{W S N}$ ' as the WSN SINR. In the simulation, we assume $W_{\text {Price }}=1.5 \mathrm{~dB}$. This is an experienced value. It is found this is the most suitable "Price" to balance the load in WSN and CN layers.

$$
\text { System }=\left\{\begin{array}{cl}
W S N, & \text { if } \operatorname{SINR}_{W S N}{ }^{\prime} \geq S I N R_{C N} \\
C N, & \text { if } \operatorname{SINR}_{W S N}{ }^{\prime}<S I N R_{C N}
\end{array}\right.
$$

The system-level simulation results are shown in Table II. The results show that the proposed scheduling scheme can provide $8.7 \%$ gain over the traditional one in average cell spectrum efficiency.

TABLE II

THE CELL SPECTRUM EFFICIENCY OF TWO SCHEDULING SCHEMES

\begin{tabular}{|c|c|c|}
\hline & $\begin{array}{c}\text { Scheme 1 } \\
\text { (Traditional) }\end{array}$ & $\begin{array}{c}\text { Scheme 2 } \\
\text { (Proposed) }\end{array}$ \\
\hline $\begin{array}{c}\text { Average cell spectrum } \\
\text { efficiency } \\
\text { (bit/s/Hz/cell) }\end{array}$ & 1.49 & 1.62 \\
\hline $\begin{array}{c}\text { Gain of Scheme 2 over } \\
\text { Scheme 1 }\end{array}$ & $0 \%$ & $8.7 \%$ \\
\hline
\end{tabular}

We can analyze the above results as following: Scheme 1 cannot achieve the balance load between WSN and CN layers. In general, over 10 terminals try to access the $\mathrm{CN}$ layer and some terminals are rejected, while the WSN layer is only partly used because only less than 10 terminals select the $\mathrm{CN}$ layer. Scheme 2 uses the "Price" weight to increase the selecting threshold for the $\mathrm{CN}$ layer. This equivalently moves some terminals from the $\mathrm{CN}$ layer to the WSN layer, and finally achieves the load balancing between the two layers.

\section{CONCLUSIONS}

This paper proposed a joint resource management scheme for a multi-layer heterogeneous the Internet of Things network. By setting a higher "Price" for the cellular network layer when "auctioning" the access resource to terminals, the values of the $\mathrm{CN}$ and WSN layers are balanced. The proposed scheme can provide a substantial capacity gain over the traditional one.

\section{ACKNOWLEDGMENT}

The research work introduced in this paper was supported by a grant from the National High Technology Research and Development Program of China (863 Program) (No. 2008AA01Z213): "Study on Heterogeneous RAN architecture and protocol based on multi-layer overlay-structuring technology".

\section{REFERENCES}

[1] V. Rodriguez, K. Moessner and R. Tafazolli, Auction Driven Dynamic Spectrum Allocation:

[2] Optimal Bidding, Pricing and Service Priorities for Multi-rate, Multiclass CDMA, IEEE PIMRC 2005, vol. 3, pp. 1850-1854, Sept. 2005.

[3] Y. Xing, R. Chandramouli and C. M. Cordeiro, Price Dynamics in Competitive Agile Spectrum Access Markets, IEEE Journal on Selected Areas in Communication, vol. 25, no. 3, pp. 613-621, Apr. 2007.

[4] J. Huang, R. A. Berry and M. L. Honig, Auction-Based Spectrum Sharing, ACM Mobile Networks and Applications J., vol. 11, no. 3, pp. 405-418, June 2006.

[5] S. Gandhi, C. Buragohain L. Cao, H. Zheng and S. Suri, A General Framework for Wireless Spectrum Auctions, IEEE International Symp. New Frontiers in Dynamic Spectrum Access Networks (DySPAN '07). 
[6] 3GPP TS 36.213 Evolved Universal Terrestrial Radio Access (EUTRA); Physical Layer Procedure (Release 8)

[7] 3GPP TS 36.211 Evolved Universal Terrestrial Radio Access (EUTRA); Physical Channels and Modulation (Release 8)
[8] ITU M.2135. Guidelines for evaluation of radio interface technologies for IMT-Advanced. 